\title{
An ultrasonic frequency sweep interferometer for liquids at high temperature:
}

\section{Mechanical assembly, signal processing, and application}

Yuhui Ai and Rebecca Lange

Department of Geological Sciences, University of Michigan, Ann Arbor, Michigan, USA

Received 2 March 2004; revised 25 June 2004; accepted 6 August 2004; published 4 December 2004.

[1] The design and application of a frequency sweep (FS) interferometer for sound speed measurements on high-temperature silicate liquids, with the potential to be applied to liquids at high pressure, are presented. FS interferometry differs from variable path length interferometry in that no mechanical movement of a buffer rod is required. Instead, a wideband pulse spanning 1-2 MHz is transmitted down the upper buffer rod, which allows maxima/minima in the returned signal to be evaluated as a periodic function of frequency. Measurements may be made at several different centered frequencies (from 3 to $7 \mathrm{MHz}$ with the transducer used in this apparatus) in order to test whether the liquid sound speed is frequency-independent and thus relaxed. This paper describes the mechanical assembly and the algorithms for signal and data processing, which are based on the solutions to the wave equations in a rod-liquid-rod interferometer. The precision and accuracy of the FS interferometer is demonstrated by measuring the relaxed sound velocities in six liquids $(\mathrm{NaCl}$, a sodium silicate, two sodium aluminosilicates, $\mathrm{CaMgSi}_{2} \mathrm{O}_{6}$, and $\mathrm{Fe}_{2} \mathrm{SiO}_{4}$ ) with well-known sound speeds from the literature. INDEX TERMS: 3994 Mineral Physics: Instruments and techniques; 3919 Mineral Physics: Equations of state; 3939 Mineral Physics: Physical thermodynamics; 3999 Mineral Physics: General or miscellaneous; 5102 Physical Properties of Rocks: Acoustic properties; KEYWORDS: frequency sweep interferometry, compressibility, silicate liquids

Citation: Ai, Y., and R. Lange (2004), An ultrasonic frequency sweep interferometer for liquids at high temperature: 2 . Mechanical assembly, signal processing, and application, J. Geophys. Res., 109, B12204, doi:10.1029/2004JB003062.

\section{Introduction}

[2] This paper is the second in a series that together describe the theoretical [Ai and Lange, 2004] (hereinafter referred to as part 1) and practical (this study) development of a new frequency sweep (FS) interferometer for the measurement of relaxed sound speeds in high-temperature liquids, with the potential to be adapted to high pressure. Measurements of relaxed sound speed, and thus compressibility, in geologically relevant liquids as a systematic function of pressure remain an experimental imperative in the earth sciences. They offer the best opportunity to obtain a comprehensive and accurate equation of state (P-V-T model) for magmatic liquids, including those containing dissolved volatile components (e.g., $\mathrm{H}_{2} \mathrm{O}$ and $\mathrm{CO}_{2}$ ). Such data are critically needed for accurate thermodynamic calculations of partial melting (and crystallization), as well as volatile degassing from melts of the deep Earth.

[3] To date, all relaxed sound velocity measurements on liquids of geological relevance have been made by variable path length (VPL) ultrasonic interferometry [e.g., Katahara et al., 1981; Rivers and Carmichael, 1987; Kress et al., 1988; Zhu et al., 1991; Secco et al., 1991; Webb and

Copyright 2004 by the American Geophysical Union. 0148-0227/04/2004JB003062\$09.00
Courtial, 1996], which requires mechanical movement of a buffer rod and thus cannot be applied at high pressure. More than 20 years ago, Katahara et al. [1981] proposed a method by which melt compressibility could be measured by varying the frequency of an ultrasonic acoustic wave through a melt, namely, frequency sweep (FS) interferometry, thus allowing measurement at high pressure. More than two decades have passed, and the FS method has not yet been applied to high-temperature silicate melts.

[4] Frequency sweep acoustic measurements have been successfully applied to high-pressure crystalline phases [e.g., Li et al., 1996, 1998; Shen et al., 1998]. However, the relatively high frequencies and broad intervals employed $(10-70 \mathrm{MHz}$ and $300-1200 \mathrm{MHz}$ ) cannot be used for relaxed measurements on silicate melts. For liquids, the frequency range must be lower $(\sim 3-10 \mathrm{MHz}$ for most silicate melts) in order for the timescale of the sound speed measurement to exceed that of structural relaxation. The challenge therefore is to perform a frequency sweep over a relatively narrow interval $(\sim 1-2 \mathrm{MHz})$ and at a relatively low centered frequency $(<10 \mathrm{MHz})$.

[5] In part 1, a theoretical acoustic model for a rodliquid-rod (RLR) interferometer and solutions to the resultant wave equations are presented. This acoustic model is the first complete theoretical analysis of the wave equations and solutions in a RLR interferometer, 


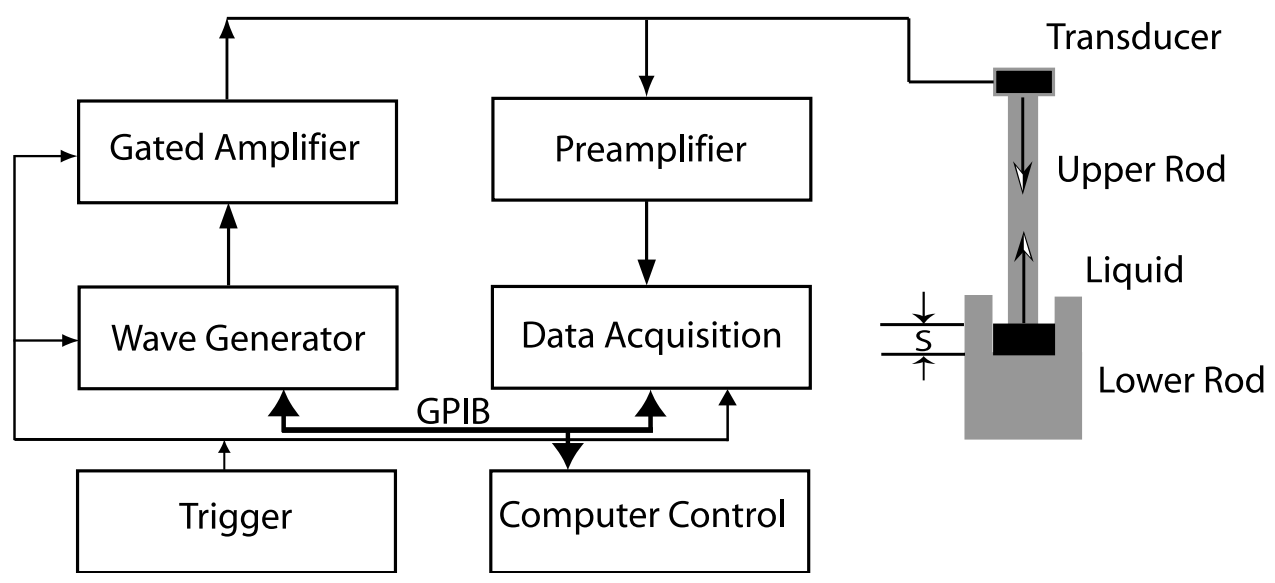

Figure 1. Schematic illustration of the frequency sweep interferometer.

which is a fundamental requirement for the successful design and construction of both the mechanical assembly and the signal processing algorithms needed to implement the new FS method applicable to silicate liquids. In this study (part 2), we describe the mechanical design and signal processing details of our FS interferometer, and we illustrate its precision and accuracy by application to six liquids ( $\mathrm{NaCl}$, a sodium silicate, two sodium aluminosilicates, $\mathrm{Fe}_{2} \mathrm{SiO}_{4}$, and $\mathrm{CaMgSi}_{2} \mathrm{O}_{6}$ ) at high temperature, which have well-documented sounds speeds in the literature. In addition, we perform both VPL and FS interferometry on a sodium silicate liquid (but with $\sim 6 \mathrm{wt} \%$ dissolved MoO; see below) to show that the two methods give similar results. The FS method has the additional advantage, however, that it can be adapted for use at high pressure in an internally heated pressure vessel.

\section{Electronic Components and Mechanical Assembly}

[6] A schematic illustration of the interferometer is shown in Figure 1. The basic design includes both an upper and lower buffer rod in the interferometric circuit, which we call the rod-liquid-rod (RLR) system. The liquid sample is contained in a cylindrical hollow $(2.4 \mathrm{~cm}$ diameter and $2.0 \mathrm{~cm}$ deep), which is drilled into a $2.8 \mathrm{~cm}$ diameter and $10 \mathrm{~cm}$ long cylindrical molybdenum rod (purity 99.999\%) that serves as the lower buffer rod. The lower rod is designed to be sufficiently long such that the round trip traveling time of the wave in the lower rod is much longer than that in the melt; this ensures that the reflections from the lower rod will not interfere with mirror reflection waves from the melt in the return signal. This eliminates the problem of reflections off the base of the melt crucible as observed in the one-rod experiments of Rivers and Carmichael [1987]. The inside base of the hollow (i.e., crucible) is well polished to reflect acoustic energy.

[7] An acoustic pulse is first programmed and formed in a computer and then its format (amplitude, frequency, duration) is delivered to an arbitrary wave generator that generates the pulse and drives the gated amplifier to produce a power output to the transducer. The transducer converts the electronic energy to an acoustic pulse that travels down to the molybdenum upper buffer rod (1.91 $\mathrm{cm}$ diameter and $40 \mathrm{~cm}$ long). When the acoustic signal reaches the rod-melt interface, part propagates into the liquid and part is reflected back up the upper rod. The part that is transmitted through the liquid reflects off the polished bottom of the crucible and undergoes multiple reflections. Part of these reflections return up the upper buffer rod, and their longitudinal parts are received by the transducer. The returned signals, which are well described by the theoretical acoustic model in part 1, are delivered to a preamplifier for amplification and then to a data acquisition system. The computer picks up the data from the data acquisition system and performs the signal processing algorithms (discussed below) to obtain the liquid sound speed. For transducer safety, the system is operated at a pulse repetition frequency of $1 \mathrm{~Hz}$.

[8] Our design differs from that used in the VPL method of Katahara et al. [1981] and Rivers and Carmichael [1987] in two critical respects. First, the initial pulse that is sent down the buffer rod is very short $(\sim 1 \mu \mathrm{s})$ compared to the pulse durations used in previous studies (e.g., $\sim 25 \mu \mathrm{s}$ [Rivers, 1985]). By significantly shortening the pulse duration, it is possible to receive the first two mirror reflections (one from the rod-liquid boundary and the other from the liquid after a round trip time) before interfering pulses (discussed by Ai and Lange [2004]) are returned up the buffer rod. Second, the initial pulse does not consist of a single frequency, but instead is a wideband pulse that encompasses a range of frequencies that span $\sim 1-2 \mathrm{MHz}$. Thus frequency is swept digitally rather than manually. This allows maxima/minima in the returned signal to be evaluated as a periodic function of frequency, which permits the sound speed to be measured via frequency sweep (FS) interferometry.

[9] A major advantage of the FS method described in this study is that it allows measurements to be made at different centered frequencies; with the transducer used in our apparatus, these may range from 3 to $7 \mathrm{MHz}$. Other transducers in our apparatus will allow a broader range of centered frequencies to be transmitted. This provides a test of whether the sound speed measured for a specific liquid at a given temperature is frequency-independent and thus relaxed. 
[10] As discussed in detail in part 1, the received signal is composed of the backward waves

$$
\begin{gathered}
w_{(e)}=\sum_{n=0}^{\infty} a_{n} s\left(t-\frac{2 n S}{c}\right)+\exp \left[j \frac{r_{m}}{a \sqrt{\left(c_{1} / c_{2}\right)^{2}-1}}\left(c_{1} t+z\right)\right] \\
\cdot w_{(A)}^{b}\left(c_{1} t+z, r\right)+\exp \left[j \frac{r_{m}}{a}\left(\sqrt{2} c_{2} t+z\right)\right] \\
\cdot w_{(B)}^{b}\left(\sqrt{2} c_{2} t+z, r\right) \\
a_{0}=R, a_{n}=R^{2 n+1}-R^{2 n-1}, n=1 \ldots \infty,
\end{gathered}
$$

where $R$ is defined in equations (44)-(45) of Ai and Lange [2004]; $w_{(A)}^{b}($ mode $\mathrm{A})$ and $w_{(B)}^{b}(\operatorname{mode} \mathrm{B})$ are pulse series with period $2 a \sqrt{\left(c_{1} / c_{2}\right)^{2}-1} / c_{1}$ and $\sqrt{2} a / c_{2}$, respectively, and with group speeds $c_{1}$ and $\sqrt{2} c_{2}$ correspondingly.

[11] In order to best resolve the mirror reflection segment from the returned signal, the constraints given in equations (2)-(5) below must be met. In these equations, $s(t)$ is the transmitted pulse, $D_{T}$ is the duration of the pulse, $c_{1}, c_{2}$ are the longitudinal and transverse wave velocities in the buffer rod material, $c$ is the liquid sound speed to be measured, $S$ is the thickness of the liquid in the RLR circuit, $a$ and $L$ are the radius and length of the upper rod, respectively, and $l$ is the length of the lower rod. All of these parameters can be varied by experimental design to optimize the delay times of the interfering pulses to best resolve the mirror reflections. In our optimally designed apparatus, $c_{1}=6250, c_{2}=3350 \mathrm{~m} / \mathrm{s}$ (those for molybdenum metal), $a=9.55 \mathrm{~mm}, L=40 \mathrm{~cm}$, $l=8 \mathrm{~cm}$. Thus the system is further optimized when

$$
\begin{gathered}
D_{T}<\tau_{1}=\frac{2 S}{c}, \\
\tau_{4}=\left|2 L\left(\frac{1}{c_{1}}-\frac{1}{\sqrt{2} c_{2}}\right)\right|>\tau_{1}+D_{T}, \\
\tau_{2}=\frac{2 a \sqrt{\left(c_{1} / c_{2}\right)^{2}-1}}{c_{1}}>\tau_{1}+D_{T}, \\
\left(\frac{2 l}{c_{1}}\right)+\frac{2 S}{c}>\tau_{1}+D_{T} .
\end{gathered}
$$

The time intervals, $\tau_{1}, \tau_{2}$, and $\tau_{4}$, are illustrated in Figure 3 of part 1. To summarize, $\tau_{1}$ is the time delay between consecutive mirror reflections, whereas $\tau_{2}$ and $\tau_{4}$, respectively, are the arrival times of the second interfering pulse of mode $\mathrm{A}$ and the first interfering pulse of mode B, respectively. The goal is to receive the first two mirror reflections before these interfering pulses arrive. Note that the first interfering pulse of mode A can be neglected because of its small magnitude, as discussed by $A i$ and Lange [2004] and more fully below with equations (8) and (9).

[12] Equation (2) restricts the duration of the initial pulse so that two separated mirror reflections can be obtained with time delay $2 S / c$ between them. In this manner, the first reflection can be separated as a reference signal sample of $s(t)$ so that its spectrum function can be calculated for deconvolution processing. Equation (2) also defines the minimum bandwidth of the initial pulse. Given that $s(t)$ is a short sine wave, the bandwidth of $s(t)$, equal to $2 / D_{T}$ in $\mathrm{Hz}$, must be wide enough to cover at least twice the frequency interval $2 \Delta f=c / S$. This minimum bandwidth allows at least two adjacent maxima and minima in the correlation function spectrum of the combination of the first two pulses to be found, from which $c$ can be reduced. In our experiments, bandwidths of $1-2 \mathrm{MHz}$ are used.

[13] According to equations (2)-(5) the melt thickness and pulse duration can be selected to optimize the experiments such that

$$
D_{T}<\frac{2 S}{c} \quad \frac{2 S}{c}+D_{T}<\frac{4 S}{c}<\tau_{2} .
$$

From equation (4) and the apparatus specifications outlined above, the time parameter $\tau_{2}$ is calculated to be $4.80 \mu \mathrm{s}$. Owing to the need for a wide bandwidth, the pulse duration is selected to be as short as possible. In this study, short CW pulses of $0.6-1.1 \mu$ s duration with frequencies ranging from 3 to $7 \mathrm{MHz}$ are attainable with the electronic components employed. As a result, if a pulse duration of $1.0 \mu \mathrm{s}$ is chosen, equation (6) becomes

$$
0.5 \mu \mathrm{s}<\frac{S}{c}<1.2 \mu \mathrm{s} .
$$

Thus the thickness of the melt layer may vary from $0.5 c$ to $1.2 c$ and thus depends on the magnitude of the sound speed of interest. For a relatively high liquid sound speed of $4000 \mathrm{~m} / \mathrm{s}$, the minimum and maximum melt thickness is 2 and $4.8 \mathrm{~mm}$, respectively, whereas for a relatively low liquid sound speed of $1500 \mathrm{~m} / \mathrm{s}$, the minimum and maximum melt thickness is 0.75 and $1.8 \mathrm{~mm}$. In contrast, if a shorter pulse duration of $0.6 \mu \mathrm{s}$ is chosen, then a broader range of melt thickness is possible, namely, $0.3 c$ to $1.2 c$. The maximum melt thickness is the same, but for a liquid sound speed of 4000 and $1500 \mathrm{~m} / \mathrm{s}$, the minimum melt thickness is 1.2 and $0.45 \mathrm{~mm}$, respectively. If attenuation in the liquid is not critical, the melt layer may be designed to be as thick as possible. For the viscosity range of most silicate liquids for which relaxed sound speeds may be obtained by ultrasonic interferometry, maximum melt thicknesses of 3-4 $\mathrm{mm}$ are recommended. The larger the melt thickness, the less error is introduced by slightly unparallel surfaces between the bottom of the upper rod and the crucible base.

\section{Signal Processing}

[14] The restrictions expressed in equations (3) and (4) allow all the interfering pulses in the upper rod, except the first pulse of mode A, to be separated and removed from $w_{(e)}$ by truncating the signal segment after a relative time $\tau_{2}$, which is equal to $2 a c_{1}^{-1} \sqrt{\left(c_{1} / c_{2}\right)^{2}-1}$ [see Ai and Lange, 2004, Figure 3]. Equation (3) indicates that a molybdenum upper rod of $40 \mathrm{~cm}$ length is long enough to separate waves of mode B from the mirror reflections, and equation (4) shows that an upper rod with a diameter of $1.91 \mathrm{~cm}$ is wide 
enough to separate interfering waves of mode A from the mirror reflections. Equation (5) ensures that echoes from a lower molybdenum rod of $8 \mathrm{~cm}$ length will not interfere with the second mirror reflection. Because the constraints in equations (2)-(5) are met by experimental design, then the following signal segment

$$
w_{s}=a_{0} s(t)+a_{1} s\left(t-\frac{2 S}{c}\right)+p_{(A)}^{b}
$$

can be extracted from $w_{(e)}$, where $p_{(A)}^{b}$ is the first interfering pulse of mode A.

[15] For buffer rods with high acoustic impedances and for short $(\sim 1 \mu \mathrm{s}) \mathrm{CW}$ pulses, the amplitude of $p_{(A)}^{b}$ is very small relative to those of the first mirror reflections from the liquid. For a molybdenum rod with diameter $1.91 \mathrm{~cm}$, the amplitude of the interfering pulse is about $25 \mathrm{~dB}$ lower than that of $a_{0} s(t)$, according to our experimental observations in part 1 [Ai and Lange, 2004, Figure 5]. Additionally, $p_{(A)}^{b}$ is always sufficiently short in duration such that no interference exists between $p_{(A)}^{b}$ and $a_{1} s[t-(2 S / c)]$. As a result, equation (8) can be approximated to the first two mirror reflections as

$$
w_{s} \approx a_{0} s(t)+a_{1} s\left(t-\frac{2 S}{c}\right) .
$$

[16] At this juncture, the correlation spectrum period finder (CSPF) algorithm is introduced, which estimates liquid sound speed from equation (9). In theory, it is similar to the amplitude spectrum method of Pialucha et al. [1989], except that our CSPF algorithm includes the implementation of deconvolution, which removes the restriction on the transmitted waveform that is otherwise required. With the first two arrivals of the mirror reflections as the signal segment to be processed, CSPF calculates the spectrum of its correlation function. Mathematically, the spectrum function of the correlation function of $w_{s}$ [Kovach, 1982] is

$$
\begin{aligned}
W_{s}(\omega) & =\frac{1}{2 \pi}\left|\int_{-\infty}^{+\infty}\left[a_{0} s(t)+a_{1} s\left(t-\frac{2 S}{c}\right)\right] e^{-j \omega t} d t\right|^{2} \\
& =\left\lfloor a_{0}^{2}+2 a_{0} a_{1} \cos \omega \frac{2 S}{c}+a_{1}^{2}\right\rfloor S^{2}(\omega),
\end{aligned}
$$

where

$$
S^{2}(\omega)=\frac{1}{2 \pi}\left|\int_{-\infty}^{+\infty} s(t) e^{-j \omega t} d t\right|^{2}
$$

is the spectrum function of the correlation function of $s(t)$.

[17] It should be noted that $S^{2}(\omega)$ is not calculated directly from the pulse that is programmed in the computer or generated in the wave generator. Owing to the complicated frequency response of the power amplifier-transducer-rod link, the waveform within the rod, as well as the one recorded in the data acquisition system, is no longer the same as that which was originally generated. Pulse edge distortion and waveform spreading are observed to have considerable impact on the estimation of $S^{2}(\omega)$. Therefore, practically, $S^{2}(\omega)$ is calculated from the first mirror reflection, which is extracted from the echo signal and is guaranteed to be a mirror reflection by equations (2)-(5).

[18] The function $F(\omega)$ is defined as the ratio of $W_{s}(\omega)$ over $S^{2}(\omega)$ :

$$
F(\omega)=\frac{W_{s}(\omega)}{S^{2}(\omega)}=\left[a_{0}^{2}+2 a_{0} a_{1} \cos \omega \frac{2 S}{c}+a_{1}^{2}\right]
$$

The invariant $\left[a_{0}^{2}+a_{1}^{2}\right]$ in equation (12) can be filtered out so that a periodic function is obtained

$$
\widetilde{F(\omega)}=2 a_{0} a_{1} \cos \omega \frac{2 S}{c} .
$$

It has period

$$
\Delta f=\frac{c}{2 S}(\mathrm{~Hz})
$$

The CSPF algorithm takes the periodic function in equation (13) and searches for a series of maxima and minima points in order to find the period $\Delta f$ in equation (14). Practically, it is an average of values of local $\Delta f$ obtained in the neighborhood of the resonance frequency of the first echo so that data with optimal signal-to-noise ratio are used. If both $\Delta f$ and $S$ (melt thickness) are known, then $c$ (liquid sound speed) is readily calculated. Although it is assumed in the CSPF algorithm that the first two reflections share an identical waveform, the algorithm is equally effective for liquids in which acoustic attenuation and waveform dispersion occur. Attenuation and dispersion only affect the phase property of the Fourier transformation of the first two echoes; therefore equation (14) stillstands, which is well documented mathematically in the work of Pialucha et al. [1989]. For relaxed liquids (which we have measured below), the experimental values of local $\Delta f$ are frequencyindependent (within error) within the limited bandwidths of $\sim 1-2 \mathrm{MHz}$, which additionally supports the validity of equation (14).

\section{Treatment of Melt Thickness $S$}

[19] In order to obtain liquid sound speed from a frequency sweep experiment, either the melt thickness or the difference in melt thickness between two experiments under otherwise identical conditions, must be known to a high accuracy and precision. In the apparatus that we have designed, the upper buffer rod is spring-loaded and attached to a micrometer, which allows the upper buffer rod to be moved up and down between experiments. The movement of the rod is recorded by the micrometer, which has a resolution better than $0.001 \mathrm{~mm}$. An estimate of melt thickness is made in the following manner. First, the buffer rod is lowered until it contacts the base of the melt crucible; this is the zero point. Then, the buffer rod is raised by the number of millimeters required for the melt thickness (e.g., $3 \mathrm{~mm}$ ). This provides a minimum estimate of $S$ owing to the fact that there is a thin film of melt between the upper rod and base of the crucible at the zero point. If the rod is then raised exactly $0.5 \mathrm{~mm}$, according to the micrometer, a much more accurate and precise measurement is made of the 
difference in melt thickness $(\Delta x=0.5 \mathrm{~mm})$ than the absolute values of melt thickness $\left(S_{1}\right.$ and $\left.S_{2}\right)$ for the two different rod positions. Because liquid sound speed is independent of melt thickness, an FS experiment performed for two different liquid depths $\left(S_{1}\right.$ and $\left.S_{2}\right)$ will lead to two different $\Delta f$ values $\left(\Delta f_{1}\right.$ and $\left.\Delta f_{2}\right)$ from equation (14). In combination, the following relationship holds:

$$
c=\frac{2 \Delta x}{\frac{1}{\Delta f_{2}}-\frac{1}{\Delta f_{1}}} .
$$

Since $S_{1}$ and $S_{2}$ are not included in equation (15) and $\Delta x$ is measured precisely, $c$ is optimally obtained from two measurements where melt thickness is varied. In practice, several different melt thickness experiments may be performed under conditions that are otherwise identical, which will allow several determinations of $c$, which can be averaged to obtain a mean and standard deviation.

[20] We note that application of FS interferometry to liquids at high pressure requires measurements for at least two different melt thicknesses without mechanical movement of the buffer rod. One possibility is to employ two upper buffer rods at the same time, which are vertically offset by a precisely known amount (e.g., $1.0 \mathrm{~mm}$ ). In order to fit two upper buffer rods into an internally heated pressure vessel (IHPV), the diameter of the rods must be reduced from that used in this study $(1.91 \mathrm{~cm})$. Fortunately, this is readily achieved by utilizing a material that has suitable longitudinal and transverse wave speeds such that $\tau_{2}$ (the arrival time of the first nonnegligible interfering pulse) is greater than sum of $D_{T}$ (the transmitted pulse duration; typically $\sim 1 \mu \mathrm{s}$ ) plus $\tau_{1}$ (the time delay between the first two mirror reflections from the liquid). For most silicate melts, $\tau_{2}$ should be greater than $4 \mu \mathrm{s}$. If alumina $\left(\mathrm{Al}_{2} \mathrm{O}_{3}\right)$ is used for the upper buffer rods, its values for $c_{1}$ and $c_{2}$ (10302 and $6173 \mathrm{~m} / \mathrm{s}$, respectively) inserted into equation (4) indicate that for $\tau_{2}=5 \mu \mathrm{s}$, an alumina upper rod radius of $\geq 1.3 \mathrm{~mm}$ is suitable. Thus two buffer rods of $5 \mathrm{~mm}$ diameter each separated by $1 \mathrm{~mm}$ have a maximum dimension of $11 \mathrm{~mm}$, which easily fits inside an internally heated pressure vessel. Similarly, the length of the upper buffer rod $(L)$ is constrained by equation (3) to be $\geq 11 \mathrm{~cm}$ (for pulse that is $1 \mu$ s long) if it is made of alumina, whereas a lower alumina $\operatorname{rod}(l)$ is constrained by equation (5) to be $\geq 0.6 \mathrm{~cm}$ (for the same $1 \mu$ s pulse duration); both of these dimensions are easily accommodated inside an internally heated pressure vessel. In order to prevent reaction between the alumina buffer rods and silicate melt, a thin coating of $\mathrm{Pt}$ can be electroplated onto the alumina rods. A thin platinum layer (e.g., $50 \mu \mathrm{m}$ ) will not interfere with the signal processing because it is much thinner than the wavelength of the acoustic wave through alumina, platinum, and silicate melt, respectively (on the millimeter scale at 4-6 MHz).

\section{Frequency Sweep Sound Speed Measurements on High-Temperature Liquids}

\subsection{NAS-8 Liquid}

[21] In order to test the FS interferometer, sound speed measurements were made on liquids with well-known sound speeds from the literature. The first liquid that we studied was a sodium aluminosilicate, sample NAS$8\left(61 \mathrm{~mol} \% \mathrm{SiO}_{2}, 6 \mathrm{~mol} \% \mathrm{Al}_{2} \mathrm{O}_{3}\right.$, and $\left.33 \mathrm{~mol} \% \mathrm{Na}_{2} \mathrm{O}\right)$, from Kress et al. [1988] at $1436^{\circ} \mathrm{C}$ and 1 bar. A series of experiments was performed on this liquid, where a fivecycle CW pulse centered at $4.5,5.0,5.5$, and $5.8 \mathrm{MHz}$ was transmitted down the buffer rod. The transmitted pulse in each experiment had a bandwidth of $1-2 \mathrm{MHz}$, with pulse durations of $1.1,1.0,0.9$, and $0.86 \mu \mathrm{s}$ long, respectively. The results and signal-processing steps for one of the experiments on this liquid (at $1436^{\circ} \mathrm{C}$ and a transmitted pulse centered at $5.0 \mathrm{MHz}$ ) are shown in Figures $2 \mathrm{a}-2 \mathrm{~d}$. In Figure $2 \mathrm{a}$, the first two returning pulses, which are the first and second mirror reflections, are shown in a plot of amplitude versus time. An estimation of the time delay between the two reflections, the two-way traveling time in the liquid, was made by measuring the distance between $b$ and $a$ in Figure 2a, the peak positions of the two oppositely polarized reflections. This time delay of $2.28 \mu \mathrm{s}$ is consistent with the frequency domain shown in Figure $2 \mathrm{~d}$, where $\Delta f$ (the difference in frequency between the minimum amplitudes at positions 2 and 1) $=440800 \mathrm{~Hz}$; thus, according to equations (2) and (14), $\tau_{1}=1 / \Delta f=2.27 \mu \mathrm{s}$, which is very close to what is observed in Figure $2 \mathrm{a}$. In Figure $2 b$, spectrum analysis of the first returning mirror reflection, the correlation function spectrum, $S^{2}(\omega)$, shows a maximum position centered at $\sim 4.8 \mathrm{MHz}$, which is shifted from the centered frequency, $5 \mathrm{MHz}$, of the transmitted pulse. This shift is caused by the complex frequency response of the gated amplifier-transducer-rod link (note that we used a $4.8 \pm 2 \mathrm{MHz}$ longitudinal transducer at $-6 \mathrm{~dB})$. It is for this reason that $S^{2}(\omega)$ is calculated in equation (11) from the first mirror reflection (shown in Figure $2 \mathrm{~b}$ ) and not from the transmitted pulse (as discussed in section 3.1). In Figure $2 c$, the correlation function spectrum of the first two mirror reflections, $W_{s}(\omega)$, is shown. Equations (12) and (13) describe how the periodic function shown in Figure $2 \mathrm{~d}$, from which $\Delta f$ is derived, is obtained from the ratio of the two functions shown in Figures $2 b$ and 2c. To convert $\Delta f$ to sound speed through equation (15), a series of experiments with different melt thicknesses were performed.

[22] For each experiment on NAS-8 liquid at $1436^{\circ} \mathrm{C}$, data were collected for five different melt thicknesses. Sound speed measurements were then obtained from equation (15) for $\Delta \mathrm{x}$ values of $1.78,1.28,1.00$, and $0.78 \mathrm{~mm}$, respectively, all of which should theoretically give the same sound speed within error. These sound speed results are summarized in Table 1. For the centered frequencies at 5.8, 5.5, 5.0, and 4.5 MHz, respectively, the average sound speeds are $2626 \pm 12,2618 \pm 16,2616 \pm 3$, and $2615 \pm$ $8 \mathrm{~m} / \mathrm{s}$. Within error, these sound speeds are frequencyindependent and indicate a relaxed liquid, which is consistent with the results of Kress et al. [1988]. Additional experiments were performed on this liquid at 1284 and $1547^{\circ} \mathrm{C}$, at a centered frequency of 5.5 and $6.0 \mathrm{MHz}$ at each temperature and for a series of melt thicknesses. The results are summarized in Table 2 . At $1284^{\circ} \mathrm{C}$, the average sound speed in NAS-8 liquid is $2726 \pm 19$ and $2729 \pm 7 \mathrm{~m} / \mathrm{s}$ for experiments with transmitted pulses centered at 5.5 and 6.0 $\mathrm{MHz}$, respectively, whereas at $1547^{\circ} \mathrm{C}$, the average sound speed is $2548 \pm 5$ and $2549 \pm 2 \mathrm{~m} / \mathrm{s}$ for the same 

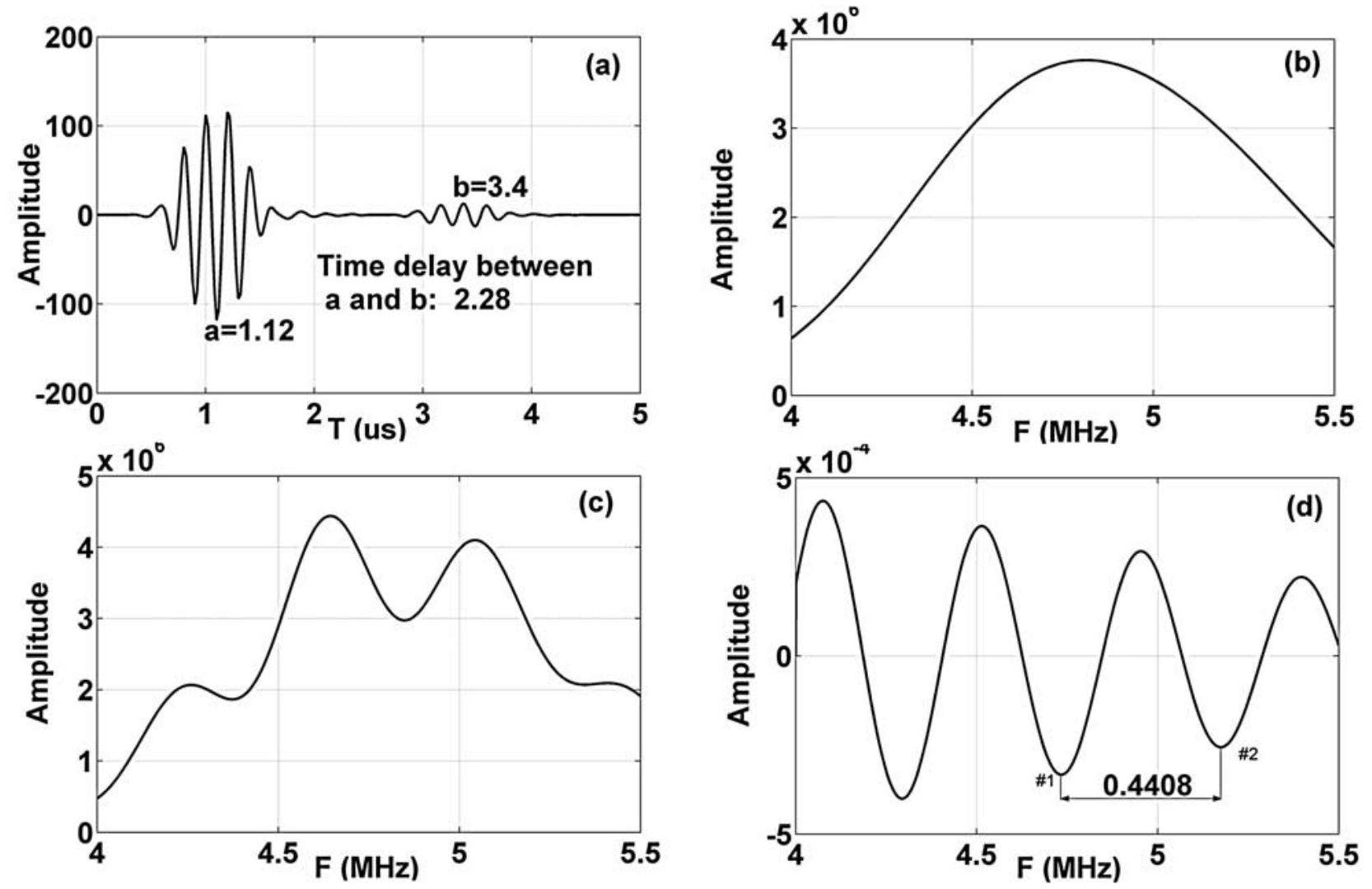

Figure 2. Experimental results on NAS-8 liquid [Kress et al., 1988] at $1436^{\circ} \mathrm{C}$ for a bandwidth of $1.5 \mathrm{MHz}$ centered at $5 \mathrm{MHz}$. Data are sampled at a rate of $50 \mathrm{MHz}$, with a frequency resolution of $100 \mathrm{~Hz}$ in the analysis of the frequency spectrum. (a) Amplitude versus time plot of the first two returning mirror reflections. (b) Amplitude versus frequency plot of the correlation function, $S^{2}(\omega)$, of the first mirror reflection. (c) Amplitude versus frequency plot of the correlation function, $W_{s}(\omega)$, of the first two mirror reflections. (d) Amplitude versus frequency plot of the periodic function, $\widetilde{F(\omega)}$, from which $\Delta f$ is obtained to derive the sound speed from equations (13)-(15).

pair of frequencies. A linear fit to these new frequency sweep sound speed data on NAS-8 liquid are within $0.1-$ $0.6 \%$ of those obtained by Kress et al. [1988] on the exact same liquid with the VPL method (Figure 3).

\subsection{NaCl Liquid}

[23] In addition to a molten silicate, experiments were also performed on a lower-density liquid, $\mathrm{NaCl}$, at 930 and $1026^{\circ} \mathrm{C}$. For each temperature, experiments were conducted at three different centered frequencies $(4.5,5.0$, and $5.8 \mathrm{MHz}$ ). Several months later, a second set of measurements was made at 849,939 , and $1026^{\circ} \mathrm{C}$; at each temperature, experiments were made at two different centered frequencies (5.8 and 4.5 MHz). The results and signalprocessing steps for the first experiment on this liquid at $1026^{\circ} \mathrm{C}$, for a transmitted pulse centered at $5.8 \mathrm{MHz}$, are shown in Figure 4. In Figure 4a, the first and second mirror reflections are shown in a plot of amplitude versus time. Because the acoustic impedance of $\mathrm{NaCl}$ liquid is less than that of NAS-8 liquid, the second reflection in Figure $4 \mathrm{a}$ is smaller than that for NAS-8 liquid in Figure 2a. In addition, the time delay between the two mirror reflections is longer in liquid $\mathrm{NaCl}$ than in liquid NAS-8. An estimation of the time delay, the distance between $b$ and $a$ in Figure 4a, gives a value of $3.280 \mu \mathrm{s}$. This time delay is consistent with the frequency domain shown in Figure $4 \mathrm{~d}$, where $\Delta f$ (the difference in frequency between the minimum amplitudes at positions 2 and 1) $=304500 \mathrm{~Hz}$; equations (2) and (14) show that $\tau_{1}=1 / \Delta f=3.284 \mu \mathrm{s}$, which is very close to what is observed in Figure 4a. In Figure 4b, the correlation function spectrum, $S^{2}(\omega)$, shows a maximum centered at $\sim 5.4 \mathrm{MHz}$, which is shifted from the centered frequency, $5.8 \mathrm{MHz}$, of the transmitted pulse. Once again, this underscores the importance of calculating $S^{2}(\omega)$ from the first mirror reflection and not from the transmitted pulse.

[24] As described above for the NAS-8 liquid, for each temperature and transmitted frequency, multiple experiments were performed on the $\mathrm{NaCl}$ liquid for different melt thicknesses in order to obtain the sound speed from equation (15). A summary of the sound speed results is given in Table 2. For the first set of experiments, at $930^{\circ} \mathrm{C}$, the average sound speeds are $1635 \pm 11,1628 \pm 10$, and $1633 \pm$ $8 \mathrm{~m} / \mathrm{s}$ for experiments with transmitted pulses centered at 5.8, 5.0, and 4.5 MHz, respectively. At $1026^{\circ} \mathrm{C}$, the sound speed values are $1548 \pm 10,1539 \pm 15$, and $1536 \pm 12 \mathrm{~m} / \mathrm{s}$, respectively, for the same set of frequencies. For the second set of experiments conducted several months later, at $849^{\circ} \mathrm{C}$, the average sound speeds are $1740 \pm 2$ and $1718 \pm$ 
Table 1. Sound Speed Data for NAS-8 Liquid at $1436^{\circ} \mathrm{C}$

\begin{tabular}{|c|c|c|c|c|c|}
\hline $\begin{array}{c}F, \\
\mathrm{MHz}\end{array}$ & $\begin{array}{l}\Delta x \\
\mathrm{~mm}\end{array}$ & $\begin{array}{c}\text { Velocity, } \\
\mathrm{m} / \mathrm{s}\end{array}$ & $\begin{array}{c}\text { Average, } \\
\mathrm{m} / \mathrm{s}\end{array}$ & $\begin{array}{l}\mathrm{SD}, \\
\mathrm{m} / \mathrm{s}\end{array}$ & $\begin{array}{c}\text { Error, } \\
\% \\
\end{array}$ \\
\hline 5.8 & 0.78 & 2637 & 2626 & 12 & 0.45 \\
\hline 5.8 & 1.00 & 2651 & & & \\
\hline 5.8 & 1.00 & 2638 & & & \\
\hline 5.8 & 1.00 & 2625 & & & \\
\hline 5.8 & 1.00 & 2620 & & & \\
\hline 5.8 & 1.00 & 2632 & & & \\
\hline 5.8 & 1.78 & 2620 & & & \\
\hline 5.8 & 1.78 & 2617 & & & \\
\hline 5.8 & 1.78 & 2616 & & & \\
\hline 5.8 & 1.78 & 2613 & & & \\
\hline 5.8 & 1.78 & 2618 & & & \\
\hline 5.5 & 0.78 & 2596 & 2618 & 16 & 0.62 \\
\hline 5.5 & 1.00 & 2634 & & & \\
\hline 5.5 & 1.00 & 2643 & & & \\
\hline 5.5 & 1.00 & 2618 & & & \\
\hline 5.5 & 1.28 & 2597 & & & \\
\hline 5.5 & 1.28 & 2599 & & & \\
\hline 5.5 & 1.28 & 2608 & & & \\
\hline 5.5 & 1.78 & 2610 & & & \\
\hline 5.5 & 1.78 & 2619 & & & \\
\hline 5.5 & 1.78 & 2617 & & & \\
\hline 5.5 & 1.78 & 2635 & & & \\
\hline 5.5 & 1.78 & 2635 & & & \\
\hline 5.0 & 1.28 & 2612 & 2616 & 3 & 0.10 \\
\hline 5.0 & 1.28 & 2615 & & & \\
\hline 5.0 & 1.78 & 2617 & & & \\
\hline 5.0 & 1.78 & 2618 & & & \\
\hline 5.0 & 1.78 & 2615 & & & \\
\hline 5.0 & 1.78 & 2619 & & & \\
\hline 4.5 & 0.78 & 2608 & 2615 & 8 & 0.31 \\
\hline 4.5 & 1.00 & 2634 & & & \\
\hline 4.5 & 1.00 & 2625 & & & \\
\hline 4.5 & 1.28 & 2605 & & & \\
\hline 4.5 & 1.28 & 2611 & & & \\
\hline 4.5 & 1.28 & 2606 & & & \\
\hline 4.5 & 1.28 & 2619 & & & \\
\hline 4.5 & 1.78 & 2616 & & & \\
\hline 4.5 & 1.78 & 2613 & & & \\
\hline 4.5 & 1.78 & 2617 & & & \\
\hline 4.5 & 1.78 & 2613 & & & \\
\hline 4.5 & 1.78 & 2613 & & & \\
\hline
\end{tabular}

Table 2. Sound Speed Data for NAS-8 and NaCl Liquids

\begin{tabular}{|c|c|c|c|c|c|}
\hline Sample & $\begin{array}{c}T, \\
{ }^{\circ} \mathrm{C}\end{array}$ & $\begin{array}{c}F, \\
\mathrm{MHz} \\
\end{array}$ & $\begin{array}{c}\text { Average } \\
\text { Velocity, } \\
\mathrm{m} / \mathrm{s}\end{array}$ & $\mathrm{SD}, \mathrm{m} / \mathrm{s}$ & Error, \% \\
\hline NAS-8 & 1284 & 6.0 & 2729 & 7 & 0.3 \\
\hline NAS-8 & & 5.5 & 2726 & 19 & 0.7 \\
\hline NAS-8 & 1436 & 5.8 & 2626 & 12 & 0.5 \\
\hline NAS-8 & & 5.5 & 2618 & 16 & 0.6 \\
\hline NAS-8 & & 5.0 & 2616 & 3 & 0.1 \\
\hline NAS-8 & & 4.5 & 2615 & 8 & 0.3 \\
\hline NAS-8 & 1547 & 6.0 & 2549 & 2 & 0.1 \\
\hline NAS-8 & & 5.5 & 2548 & 5 & 0.2 \\
\hline $\mathrm{NaCl}(2)$ & 849 & 5.8 & 1740 & 2 & 0.1 \\
\hline $\mathrm{NaCl}(2)$ & & 4.5 & 1718 & 5 & 0.3 \\
\hline $\mathrm{NaCl}$ (1) & 930 & 5.8 & 1635 & 11 & 0.6 \\
\hline $\mathrm{NaCl}$ (1) & & 5.0 & 1628 & 10 & 0.6 \\
\hline $\mathrm{NaCl}$ (1) & & 4.5 & 1633 & 8 & 0.5 \\
\hline $\mathrm{NaCl}(2)$ & 939 & 5.8 & 1639 & 8 & 0.5 \\
\hline $\mathrm{NaCl}(2)$ & & 4.5 & 1646 & 2 & 0.1 \\
\hline $\mathrm{NaCl}$ (1) & 1026 & 5.8 & 1548 & 10 & 0.6 \\
\hline $\mathrm{NaCl}(2)$ & & 5.8 & 1579 & 2 & 0.1 \\
\hline $\mathrm{NaCl}$ (1) & & 5.0 & 1539 & 15 & 0.9 \\
\hline $\mathrm{NaCl}$ (1) & & 4.5 & 1536 & 12 & 0.7 \\
\hline $\mathrm{NaCl}(2)$ & & 4.5 & 1560 & 2 & 0.1 \\
\hline
\end{tabular}

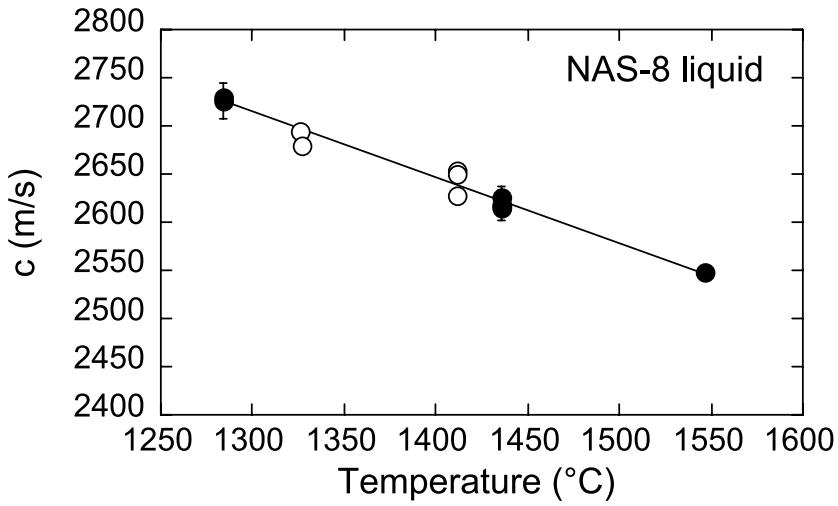

Figure 3. Plot of sound speed measurements versus temperature for NAS- 8 liquid at 1 bar. Solid circles are from this study obtained by the FS method, whereas open circles are from Kress et al. [1988] obtained by VPL method. Errors shown from this study are one standard deviation. The solid line is a linear fit to the data in this study and matches the measurements of Kress et al. [1988] within $0.1-0.6 \%$.

$5 \mathrm{~m} / \mathrm{s}$ at 5.8 and $4.5 \mathrm{MHz}$, respectively. At $939^{\circ} \mathrm{C}$, the sound speeds are $1639 \pm 8$ and $1646 \pm 2 \mathrm{~m} / \mathrm{s}$ at 5.8 and 4.5 $\mathrm{MHz}$, respectively. Finally, at $1026^{\circ} \mathrm{C}$, the sound speeds are $1579 \pm 2$ and $1560 \pm 2 \mathrm{~m} / \mathrm{s}$ at 5.8 and $4.5 \mathrm{MHz}$, respectively. These results are shown in Figure 5 and compared to those obtained by Kress et al. [1988] using the VPL method. Our results are within +1.8 to $-1.7 \%$ (or $\pm 30 \mathrm{~m} / \mathrm{s}$ ) of the linear fit to the data of Kress et al. [1988], with an average deviation of $\pm 0.9 \%$ (or \pm $14 \mathrm{~m} / \mathrm{s}$ ). Our sound speed data for $\mathrm{NaCl}$ liquid have more scatter than our results on silicate liquids (see below), which is probably a result of the lower acoustic impedance of $\mathrm{NaCl}$ liquid compared to silicate liquids. The greater mismatch in acoustic impedance between the molybdenum rod and $\mathrm{NaCl}$ liquid leads to a smaller amplitude for the second mirror reflection and thus a smaller signal-to-noise ratio.

\subsection{Three Additional Silicate Liquids: NAS-15, $\mathrm{CaMgSi}_{2} \mathrm{O}_{6}$, and $\mathrm{Fe}_{2} \mathrm{SiO}_{4}$}

[25] In addition to the NAS-8 and $\mathrm{NaCl}$ liquids, we also measured sound speeds in three other silicate liquids, which can be compared to previous measurements from the literature. The first is another sodium aluminosilicate liquid from Kress et al. [1988], NAS-15 (52.4 mol \% $\mathrm{SiO}_{2}, 15.2 \mathrm{~mol} \% \mathrm{Al}_{2} \mathrm{O}_{3}$, and $32.4 \mathrm{~mol} \% \mathrm{Na}_{2} \mathrm{O}$ ), whereas the other two are $\mathrm{Fe}_{2} \mathrm{SiO}_{4}$ (fayalite) liquid and $\mathrm{CaMgSi}_{2} \mathrm{O}_{6}$ (diopside) liquid, which were previously studied by Rivers and Carmichael [1987]. The results are tabulated in Table 3 and shown in Figures 6, 7, and 8. Our measurements are shown as solid circles, whereas those from the literature are open circles. For each liquid, we significantly extended the temperature interval of the experimental data.

[26] For example, Kress et al. [1988] present relaxed data at a single temperature only $\left(1418^{\circ} \mathrm{C}\right)$ for NAS-15 liquid, whereas we have extended relaxed measurements to $1613^{\circ} \mathrm{C}$. We were unable to obtain a relaxed measurement at $\sim 1400^{\circ} \mathrm{C}$; however, our results at 1516,1586 , and 

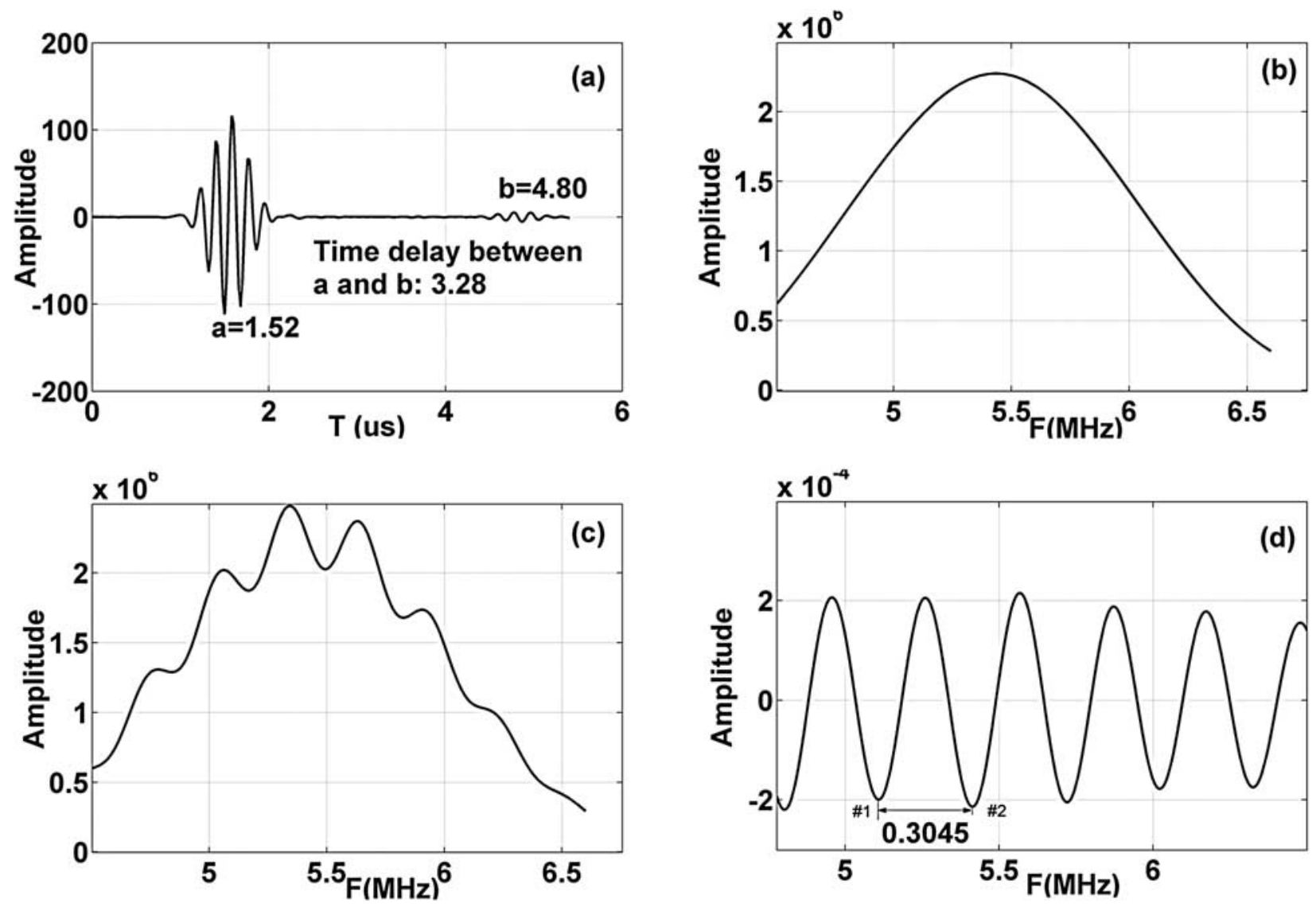

Figure 4. Experimental results on $\mathrm{NaCl}$ liquid at $1026^{\circ} \mathrm{C}$ for a bandwidth of $1.6 \mathrm{MHz}$ centered at $5.8 \mathrm{MHz}$. Data are sampled at a rate of $50 \mathrm{MHz}$, with a frequency resolution of $100 \mathrm{~Hz}$ in the analysis of the frequency spectrum. (a) Amplitude versus time plot of the first two returning mirror reflections. (b) Amplitude versus frequency plot of the correlation function, $S^{2}(\omega)$, of the first mirror reflection. (c) Amplitude versus frequency plot of the correlation function, $W_{s}(\omega)$, of the first two mirror reflections. (d) Amplitude versus frequency plot of the periodic function, $\widetilde{F(\omega)}$, from which $\Delta f$ is obtained to derive the sound speed from equations (13)-(15).

$1613^{\circ} \mathrm{C}$ are fully consistent with the relaxed measurement of Kress et al. [1988] at $1418^{\circ} \mathrm{C}$ (Figure 6). The only discrepant data point (by $1.1 \%$ ) is our measurement at $4 \mathrm{MHz}$ and $1516^{\circ} \mathrm{C}$, which is unlikely to be a problem with relaxation because the measurement at $5 \mathrm{MHz}$ and $1516^{\circ} \mathrm{C}$ is within $0.2 \%$ of a linear fit to all the data. If the $5 \mathrm{MHz}$ sound speed measurement is relaxed, then so must be the lower-frequency measurement.

[27] As another example, we measured sound speeds in $\mathrm{Fe}_{2} \mathrm{SiO}_{4}$ (fayalite) liquid over a $386^{\circ}$ interval and compare our results to those of Rivers and Carmichael [1987] over a $150^{\circ}$ interval in Figure 7 . The two data sets are within $0.5 \%$ of each other, which indicates excellent interlaboratory agreement. Additionally, in Figure 8 and Table 3, we present new sound speed measurements on $\mathrm{CaMgSi}_{2} \mathrm{O}_{6}$ liquid over a $190^{\circ}$ interval, which allows the negative temperature dependence of the sound speed for this liquid to be resolved. Our results are within 0.1$1.1 \%$ of those measured by Rivers and Carmichael [1987] at 1425 and $1485^{\circ} \mathrm{C}$. The general conclusion is that relaxed liquid sound speeds can be measured in hightemperature silicate liquids with our new FS interferom-

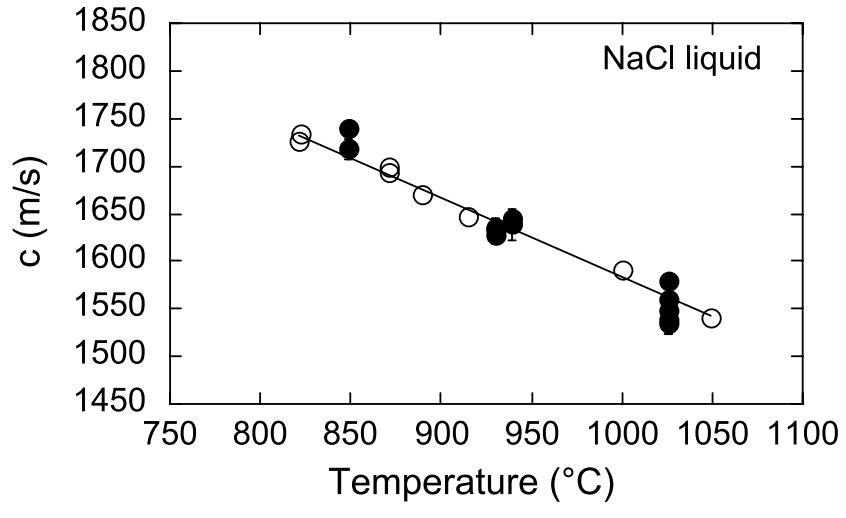

Figure 5. Plot of sound speed measurements versus temperature for $\mathrm{NaCl}$ liquid at 1 bar. Solid circles are from this study obtained by the FS method, whereas open circles are from Kress et al. [1988] obtained by VPL method. Errors shown from this study are one standard deviation. The solid line is a linear fit to the data from Kress et al. [1988]. Our measurements are within +1.8 to $-1.7 \%$ to this fitted line, with an average deviation of $\pm 0.9 \%$ (or $\pm 14 \mathrm{~m} / \mathrm{s}$ ). 
Table 3. Sound Speeds for NAS-15, $\mathrm{Fe}_{2} \mathrm{SiO}_{4}, \mathrm{CaMgSi}_{2} \mathrm{O}_{6}$, and NS-A Liquids

\begin{tabular}{|c|c|c|c|c|c|}
\hline Sample & $\begin{array}{c}T, \\
{ }^{\circ} \mathrm{C} \\
\end{array}$ & $\begin{array}{c}F, \\
\mathrm{MHz}\end{array}$ & $\begin{array}{c}\text { Average } \\
\text { Velocity, } \\
\mathrm{m} / \mathrm{s}\end{array}$ & $\mathrm{SD}, \mathrm{m} / \mathrm{s}$ & Error, \% \\
\hline NAS-15 & 1516 & 5.0 & 2782 & 4 & 0.1 \\
\hline NAS-15 & & 4.0 & 2814 & 8 & 0.3 \\
\hline NAS-15 & 1586 & 4.5 & 2725 & 1 & 0.1 \\
\hline NAS-15 & 1613 & 6.0 & 2716 & 8 & 0.3 \\
\hline NAS-15 & & 4.5 & 2705 & 1 & 0.1 \\
\hline $\mathrm{Fe}_{2} \mathrm{SiO}_{4}$ & 1243 & 6.0 & 2460 & 7 & 0.3 \\
\hline $\mathrm{Fe}_{2} \mathrm{SiO}_{4}$ & & 4.5 & 2466 & 1 & 0.1 \\
\hline $\mathrm{Fe}_{2} \mathrm{SiO}_{4}$ & 1343 & 6.0 & 2405 & 2 & 0.1 \\
\hline $\mathrm{Fe}_{2} \mathrm{SiO}_{4}$ & & 4.5 & 2414 & 4 & 0.2 \\
\hline $\mathrm{Fe}_{2} \mathrm{SiO}_{4}$ & 1348 & 5.0 & 2419 & 5 & 0.2 \\
\hline $\mathrm{Fe}_{2} \mathrm{SiO}_{4}$ & & 4.5 & 2421 & 7 & 0.3 \\
\hline $\mathrm{Fe}_{2} \mathrm{SiO}_{4}$ & 1490 & 6.0 & 2387 & 2 & 0.1 \\
\hline $\mathrm{Fe}_{2} \mathrm{SiO}_{4}$ & & 4.5 & 2392 & 3 & 0.1 \\
\hline $\mathrm{Fe}_{2} \mathrm{SiO}_{4}$ & 1606 & 6.0 & 2332 & 2 & 0.1 \\
\hline $\mathrm{Fe}_{2} \mathrm{SiO}_{4}$ & & 4.5 & 2326 & 7 & 0.3 \\
\hline $\mathrm{CaMgSi}_{2} \mathrm{O}_{6}$ & 1426 & 6.0 & 3035 & 5 & 0.2 \\
\hline $\mathrm{CaMgSi}_{2} \mathrm{O}_{6}$ & & 4.5 & 3051 & 5 & 0.3 \\
\hline $\mathrm{CaMgSi}_{2} \mathrm{O}_{6}$ & 1530 & 6.0 & 3015 & 8 & 0.5 \\
\hline $\mathrm{CaMgSi}_{2} \mathrm{O}_{6}$ & & 4.5 & 3012 & 6 & 0.4 \\
\hline $\mathrm{CaMgSi}_{2} \mathrm{O}_{6}$ & 1614 & 6.0 & 3001 & 10 & 0.7 \\
\hline $\mathrm{CaMgSi}_{2} \mathrm{O}_{6}$ & & 4.5 & 2989 & 8 & 0.3 \\
\hline NS-A & 1205 & 6.0 & 2677 & 3 & 0.1 \\
\hline NS-A & & 4.5 & 2680 & 6 & 0.2 \\
\hline NS-A & 1304 & 6.0 & 2598 & 6 & 0.2 \\
\hline NS-A & & 4.5 & 2592 & 8 & 0.3 \\
\hline NS-A & 1404 & 6.0 & 2494 & 7 & 0.3 \\
\hline NS-A & & 4.5 & 2503 & 9 & 0.4 \\
\hline
\end{tabular}

eter with an accuracy and precision that is often $\leq 1 \%$ relative.

\section{Comparison of FS and VPL Sound Speed Measurements From This Study}

[28] The experimental apparatus presented in this study is readily adapted to measure sound speed by the VPL method; it is not restricted to the FS method. As previously discussed in part 1 , the entire echo signal returned from a RLR interferometer is characterized by the reflection coef-

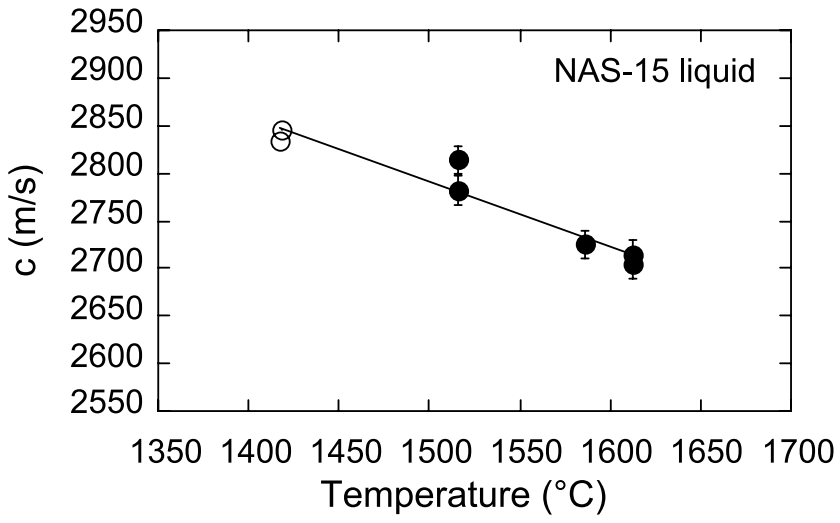

Figure 6. Plot of sound speed measurements versus temperature for NAS-15 liquid at 1 bar. Solid circles are from this study obtained by the FS method, whereas open circles are from Kress et al. [1988] obtained by VPL method. Errors shown from this study are one standard deviation.

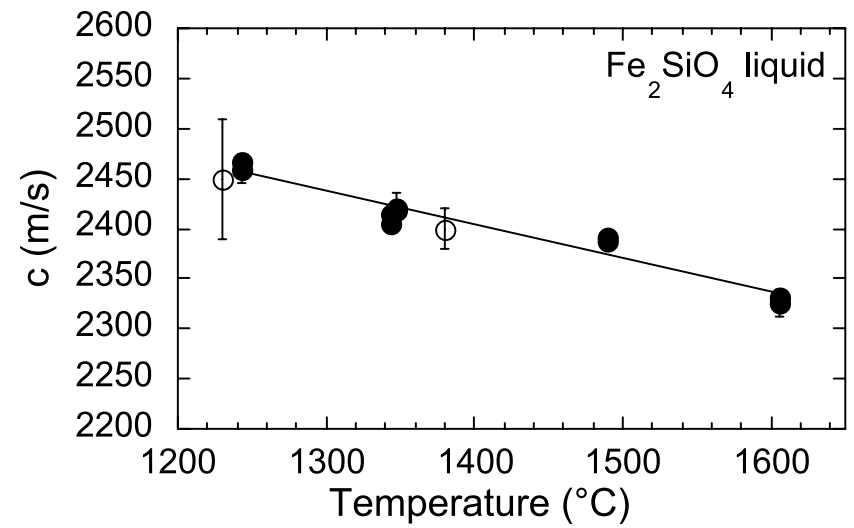

Figure 7. Plot of sound speed measurements versus temperature for $\mathrm{Fe}_{2} \mathrm{SiO}_{4}$ liquid at 1 bar. Solid circles are from this study obtained by the FS method, whereas open circles are from Rivers and Carmichael [1987] obtained by VPL method. Errors shown from this study are one standard deviation.

ficients, $\beta_{(i, A)}, \beta_{(i, B)}$ and $\beta_{A}$. Each of these reflection coefficients is a periodic function of $S$, the liquid thickness, as illustrated in equations (30), (33), and (34) of Ai and Lange [2004]. Therefore they all have identical period, $\Delta S=c / 2 f$, where $f$ is the transmitted frequency of the acoustic pulse and $c$ is the sound speed. This result shows that a change in path length, $S$, will lead to a periodic change in the echo amplitude with maxima/minima that are spaced with the same interval, $\Delta S$. This is the basis for the VPL method used in the literature. As shown below, our system does not require any knurling of the upper buffer rod to eliminate interfering pulses in the measurement of sound speed by the VPL method, in contrast to previous studies [e.g., Rivers, 1985]. Because of the more complete theoretical treatment in part 1 , in which the full wave equations in the RLR configuration are solved, the interfering pulses are demonstrated to change periodically with melt thickness; therefore

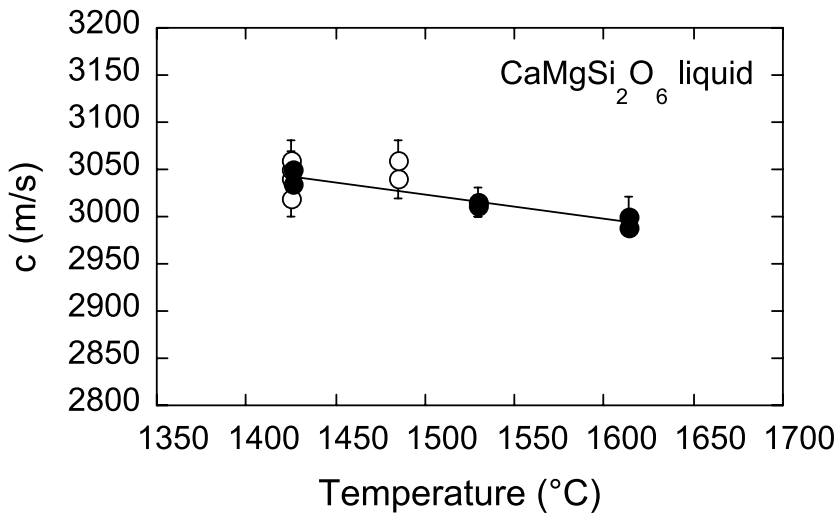

Figure 8. Plot of sound speed measurements versus temperature for $\mathrm{CaMgSi}_{2} \mathrm{O}_{6}$ liquid at 1 bar. Solid circles are from this study obtained by the FS method, whereas open circles are from Rivers and Carmichael [1987] obtained by VPL method. Errors shown from this study are one standard deviation. 


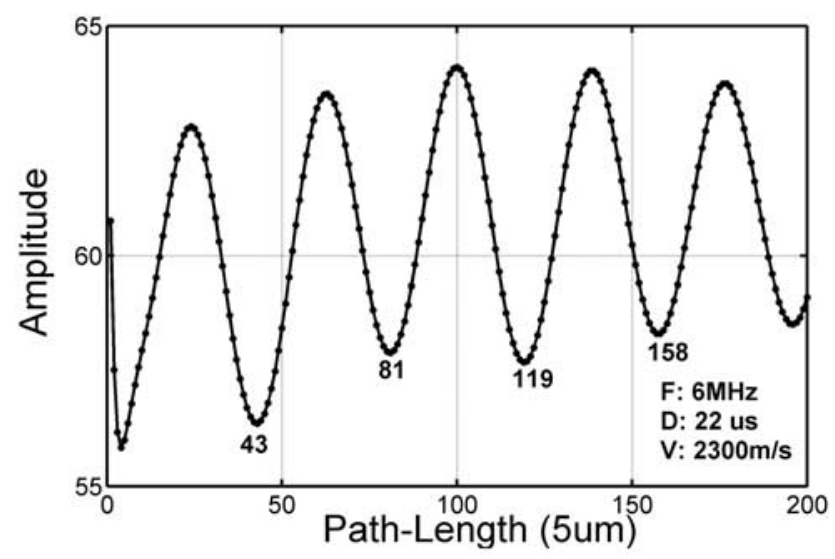

Figure 9. Plot of amplitude versus path length for liquid NS-A liquid (with 6 wt \% MoO) at $1444^{\circ} \mathrm{C}$ obtained with the VPL method in this study. The transmitted frequency was $6 \mathrm{MHz}$ with a pulse duration of $22 \mu \mathrm{m}$. Data were obtained along path length increments of $0.005 \mathrm{~mm}$.

there is no need to eliminate them in order to perform a VPL measurement of sound speed.

[29] As a further verification of both the acoustic model in part 1 and the experimental apparatus described in this study, we provide a comparison between the VPL and FS method for sound speed measurements on the same liquid. In this case, we are comparing two different methods utilizing the same mechanical assembly and electronic components. Application of the VPL method requires that reflections from the base of the crucible (in our case the lower rod serves as the crucible; Figure 1) do not interfere with the received signal. For our system, this means that the $\mathrm{CW}$ pulse transmitted down the upper rod must be shorter than $25 \mu$ s duration.

[30] Figure 9 presents the results of a VPL experiment that we performed at $1444^{\circ} \mathrm{C}$ on a sodium silicate liquid (NS-A from Kress et al. [1988] but with $6 \mathrm{wt} \%$ dissolved $\mathrm{MoO}$ owing to prolonged time in the Mo crucible at high temperatures as discussed more fully below) with a $22 \mu \mathrm{s}$ pulse at a fixed frequency of $6 \mathrm{MHz}$. Path length (or melt thickness) was varied by moving the spring-loaded buffer rod that is attached to a digital micrometer. Data were collected along 5- $\mu \mathrm{m}$ increments as a function of melt thickness. The amplitude of the echo signal, fitted to a polynomial, versus path length is shown in Figure 9. The minima are marked (positions 43, 81, 119, and 158) and the average $\Delta S$ value between adjacent minima is $191.7 \pm$ $2.5 \mu \mathrm{m}$, which gives a sound speed of $2300 \pm 30 \mathrm{~m} / \mathrm{s}$ at

Table 4. VPL and FS Sound Speed Data on NS Liquid With $6 \mathrm{wt} \% \mathrm{MoO}$

\begin{tabular}{lllclc}
\hline & & & & & \multicolumn{2}{c}{ Micrometer Step, } \\
$T,{ }^{\circ} \mathrm{C}$ & $F, \mathrm{MHz}$ & $\mathrm{FS} c, \mathrm{~m} / \mathrm{s}$ & Error, \% & VPL $c, \mathrm{~m} / \mathrm{s}$ & $\mu \mathrm{m}$ \\
\hline 1284 & 6.0 & $2459 \pm 9$ & 0.36 & $2420 \pm 30$ & 5 \\
& 5.5 & $2467 \pm 10$ & 0.42 & - & \\
1444 & 6.0 & $2327 \pm 6$ & 0.26 & $2300 \pm 30$ & 5 \\
& 5.5 & $2287 \pm 10$ & 0.42 & - & \\
1547 & 6.0 & $2182 \pm 8$ & 0.40 & - & \\
& 5.5 & $2205 \pm 14$ & 0.65 & - & \\
\hline
\end{tabular}

$6 \mathrm{MHz}$. A similar VPL experiment was performed on this sodium silicate liquid at $1284^{\circ} \mathrm{C}$ and $6 \mathrm{MHz}$, giving rise to a sound speed of $2420 \pm 30 \mathrm{~m} / \mathrm{s}$ (Table 4 ).

[31] After completing the VPL measurements, sound speeds were then measured on this same liquid sample with the FS method at 1284,1444 , and $1547^{\circ} \mathrm{C}$. At each temperature, data were collected for various melt thicknesses at two centered frequencies, 5.5 and $6.0 \mathrm{MHz}$. A comparison of the FS and VPL data sets are given in Table 4 and Figure 10. The results show that the two methods give nearly identical results, with an experimental precision that is typically $\leq 0.7 \%$ for the FS method and $\leq 1.3 \%$ for the VPL method.

\subsection{Mo Contamination of Highly Alkaline, Binary Silicate Melts}

[32] The sound speeds measured for the sodium silicate liquid in this study are significantly lower than those reported for this same liquid composition by Kress et al. [1988] (their sample NS-A). The reason for the discrepancy is that this highly sodic liquid $\left(\sim 40\right.$ wt $\left.\% \mathrm{Na}_{2} \mathrm{O}\right)$ dissolved considerable amounts of molybdenum (6.0 wt $\% \mathrm{MoO}$ or $3.4 \mathrm{~mol} \% \mathrm{MoO}$ ) from the crucible in our study, owing to a prolonged residence ( 2 days) in the Mo crucible at high temperature (although taken to room temperature overnight) prior to data collection. In contrast, the sodium aluminosilicate liquid (NAS-8) in this study was much more resistant to Mo dissolution, and we detected $<0.2 \mathrm{wt} \% \mathrm{MoO}$ in our postexperimental glass. The long time period that the NS sample was held at high temperature is not a requirement of our system, and the only reason it occurred was to perform a check and calibration of the electronic components prior to data collection. In fact, it is possible to perform the FS and VPL experiments rapidly. For example, a series of twenty FS experiments at a given temperature (e.g., five melt thickness experiments for each of four different transmitted frequencies) takes less than $30 \mathrm{~min}$ to complete.

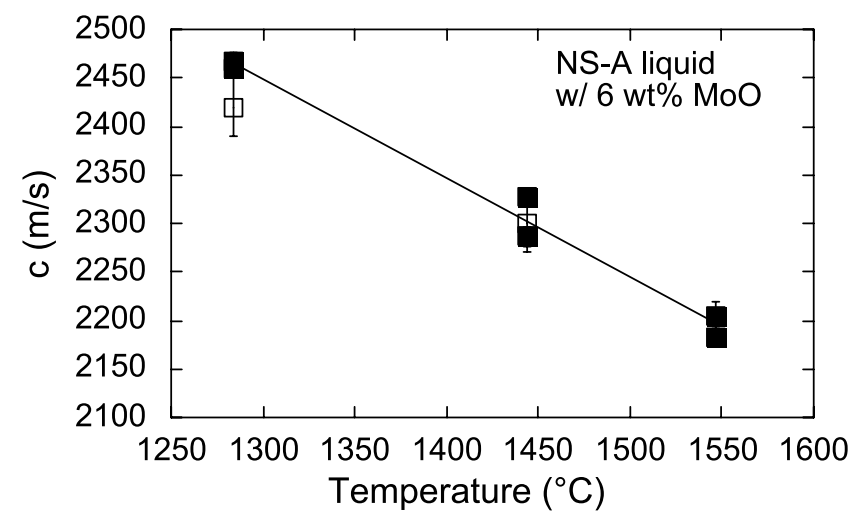

Figure 10. Plot of sound speed measurements versus temperature for NS-A liquid (with $6 \mathrm{wt} \% \mathrm{MoO}$ ) at 1 bar. Solid squares are from this study obtained by the FS method, whereas open squares are from this study obtained by VPL method. The solid line is a linear fit to the FS data. The data demonstrate agreement within one standard deviation between the FS and VPL method for sound speed measurements in liquids at high temperatures and 1 bar. 


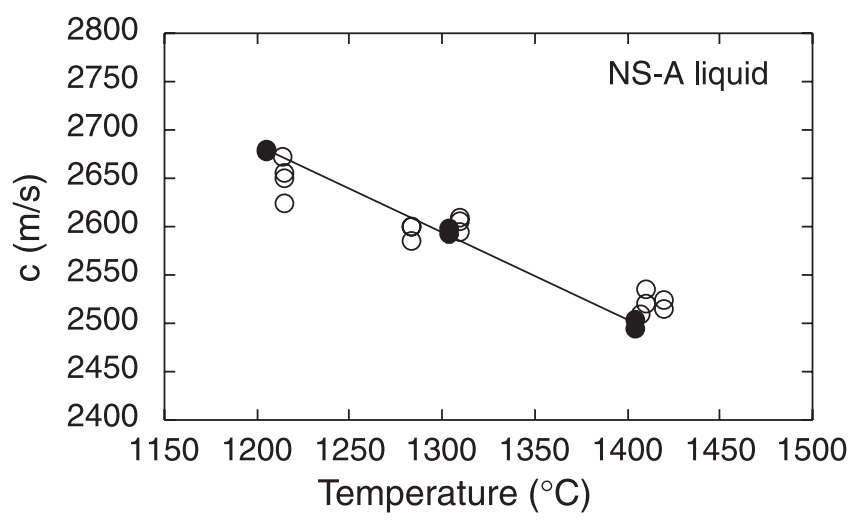

Figure 11. Plot of sound speed measurements versus temperature for NS-A liquid (with $<0.3 \mathrm{wt} \% \mathrm{MoO}$ ) at $1 \mathrm{bar}$. Solid circles are from this study obtained by the FS method, whereas open circles are from Kress et al. [1988] obtained by VPL method. Errors shown from this study are one standard deviation.

[33] Our results with the NS liquid underscore the importance of monitoring for Mo contamination in the sample melts and taking steps to minimize it. Alkali-rich, binary silicate melts are especially problematic. As another example, Rivers and Carmichael [1987] showed that up to $4.7 \mathrm{~mol} \% \mathrm{MoO}$ contaminated one of their binary silicate liquids with $26 \mathrm{~mol} \% \mathrm{~K}_{2} \mathrm{O}$, whereas their $\mathrm{CaMgSiO}_{3}$ and $\mathrm{MgSiO}_{3}$ liquids dissolved only 0.77 and $0.03 \mathrm{~mol} \% \mathrm{MoO}$, respectively. Despite the problems of Mo contamination in the highly sodic, binary silicate melt, our results in Table 4 and Figures 9 and 10 confirm the internal consistency between the FS and VPL methods with our newly designed acoustic interferometer. The success of both techniques, without requirement of any knurling of the upper rod, further demonstrates the validity of the theoretical acoustic model presented in part 1 , which provides the foundation for the experimental apparatus and signal processing presented in this study.

\subsection{A Second Set of Sound Speed Measurements on NS-A Liquid}

[34] In order to confirm that the discrepancy between our first set of sound speed measurements on NS-A liquid and those of Kress et al. [1988] is due to MoO contamination, we performed a second set of experiments using the FS method on a fresh sample. In this case, the liquid was held at high temperature for less than 5 hours. Moreover, we did not take the sample above $1405^{\circ} \mathrm{C}$, whereas we previously performed measurements up to $1547^{\circ} \mathrm{C}$. Sound speeds were measured at 1205,1304 , and $1405^{\circ} \mathrm{C}$; at each temperature, data were collected at 6 and $4.5 \mathrm{MHz}$ (Table 3). The results are plotted as a function of temperature in Figure 11, and compared to the sound speed data of Kress et al. [1988] for this exact same liquid. Overall, there is good agreement, although the data of Kress et al. [1988] are more scattered than our measurements; they range from +1.7 to $-1.6 \%$ of the linear fit to our new measurements, with an average deviation of $0.9 \%$. It is unlikely that this scatter is a result of Mo contamination, because the Kress et al. [1988] measurements at $1410^{\circ} \mathrm{C}$ (runs 1 and 2) contained $<0.26 \mathrm{~mol} \%$ $\mathrm{MoO}_{3}$, whereas those at $1420^{\circ} \mathrm{C}$ (runs $12-15$ ) contained $\sim 0.46 \mathrm{~mol} \% \mathrm{MoO}_{3}$; thus the first few runs and the last few runs were made by Kress et al. [1988] at nearly the same temperature and show good agreement (within 0.4\%) despite the small increase in $\mathrm{MoO}_{3}$.

[35] The comparison between our data set and that of Kress et al. [1988] confirms that our new FS acoustic interferometer provides results that are in agreement with measurements from the literature obtained by the VPL method. On the basis of these comparisons, the accuracy of our sound speed measurements on high-temperature silicate liquids is generally better than $1 \%$ relative.

\section{Further Applications}

[36] A primary motivation for the development of this frequency sweep interferometer is to extend relaxed sound speed measurements on silicate liquids to high pressure in an internally heated pressure vessel. In a future contribution, we will present both the theoretical and practical development of a FS interferometer applicable to liquids at high pressure. In the meantime, there is a considerable need to expand 1-bar sound speed measurements on geologically relevant liquids over a much wider range of temperature and composition. Currently, the temperature interval over which most relaxed sound speeds have been measured in individual liquids is relatively narrow, typically spanning only $\sim 50-150^{\circ}$ [e.g., Rivers and Carmichael, 1987; Kress et al., 1988; Webb and Courtial, 1996], with most measurements between 1350 and $1500^{\circ} \mathrm{C}$. Because sound speed is measured with an error of $\sim 1 \%$ on average, a propagation of errors indicates that measurements over a $100-200^{\circ}$ interval will lead to uncertainties in $d c / d T$ of $\sim 50-100 \%$. As a consequence, extrapolation outside the temperature range of sound speed measurements (e.g., to the $700-1000^{\circ} \mathrm{C}$ range of siliceous, crustal melts or the $>1600^{\circ} \mathrm{C}$ range of high-pressure, highmagnesium melts) is subject to significant error.

[37] The FS apparatus described in this paper can be used to expand the temperature interval over which silicate melt sound speeds are measured. Examples are illustrated in this study, where we have doubled and tripled the temperature range of relaxed sound speed measurements for various silicate liquids. We plan to use this FS interferometer in an ultrahigh-temperature furnace (to $2000^{\circ} \mathrm{C}$ ) to greatly extend both the temperature and compositional range of the sound speed data set for silicate liquids at one bar.

[38] Acknowledgments. This research was supported by the National Science Foundation (EAR-0087764) and seed funds from the University of Michigan. Insightful comments by Victor Kress, Baosheng Li, Mark Rivers, Ian Carmichael, and Ian Jackson substantially improved this manuscript.

\section{References}

Ai, Y., and R. A. Lange (2004), An ultrasonic frequency sweep interferometer for liquids at high temperature: 1. Acoustic model, J. Geophys. Res., B12203, doi:10.1029/2003JB002842.

Katahara, K. W., C. S. Rai, M. H. Manghnani, and J. Balogh (1981), An interferometric technique for measuring velocity and attenuation in molten rocks, J. Geophys. Res., 86, 11,779-11,786.

Kovach, L. D. (1982), Advanced Engineering Mathematics, 706 pp., Addision-Wesley-Longman, Reading, Mass.

Kress, V. C., Q. Williams, and I. S. E. Carmichael (1988), Ultrasonic investigation of melts in the system $\mathrm{Na}_{2} \mathrm{O}-\mathrm{Al}_{2} \mathrm{O}_{3}-\mathrm{SiO}_{2}$, Geochim. Cosmochim. Acta, 52, 283-293.

Li, B., I. Jackson, T. Gasparik, and R. C. Liebermann (1996), Elastic wave velocity measurement in multi-anvil apparatus to $10 \mathrm{GPa}$ using ultrasonic interferometry, Phys. Earth Planet. Inter., 98, 79-91. 
Li, B., G. Chen, D. G. Gwanmesia, and R. C. Liebermann (1998), Sound velocity measurement at mantle transition zone conditions of pressure and temperature using ultrasonic interferometery in a multianvil apparatus, in Properties of Earth and Planetary Materials at High Pressure and Temperature, Geophys. Monogr. Ser., vol. 101, edited by H. M. Murli and Y. Takehiko, pp. 35-40, AGU, Washington, D. C.

Pialucha, T., C. C. H. Guyott, and P. Cawley (1989), Amplitude spectrum method for the measurement of phase velocity, Ultrasonics, 27, 270-279.

Rivers, M. L. (1985), Ultrasonic studies of silicate melts, Ph.D. thesis, Univ. of Calif., Berkeley.

Rivers, M. L., and I. S. E. Carmichael (1987), Ultrasonic studies of silicates melt, J. Geophys. Res., 92, 9247-9270.

Secco, R. A., M. H. Manghnani, and T. C. Liu (1991), Velocities and compressibility of komatiitic melts, Geophys. Res. Lett., 18, 1397-1400.

Shen, A. H., H.-J. Reichmann, G. Chen, R. J. Angel, W. A. Bassett, and H. Spetzler (1998), GHz ultrasonic interferometry in a diamond anvil cell: P-wave velocity in periclase to $4.4 \mathrm{GPa}$ and $207^{\circ} \mathrm{C}$, in Properties of Earth and Planetary Materials at High Pressure and Temperature, Geophys. Monogr. Ser., vol. 101, edited by M. H. Manghnani and Y. Takehiko, pp. 71-77, AGU, Washington, D. C

Webb, S. L., and P. Courtial (1996), Compressibility of melts in the CaO$\mathrm{Al}_{2} \mathrm{O}_{3}-\mathrm{SiO}_{2}$ system, Geochim. Cosmochim. Acta, 60, 75-86.

Zhu, H., T. Saito, Y. Sato, T. Yamamura, K. Shimakage, and T. Ejima (1991), Ultrasonic velocity and absorption coefficient in molten alkali metal nitrates and carbonates, J. Jpn. Inst. Metals, 55, 937-944.

Y. Ai and R. Lange, Department of Geological Sciences, 2534 C.C. Little Building, University of Michigan, Ann Arbor, MI 48109-1063, USA. (yuhui@umich.edu; becky@umich.edu) 